\title{
The oligosaccharide $\alpha$-cyclodextrin has modest effects to slow gastric emptying and modify the glycaemic response to sucrose in healthy older adults
}

\author{
Diana Gentilcore ${ }^{1}$, Lora Vanis ${ }^{1,2}$, Jasmine C. Teng ${ }^{1}$, Judith M. Wishart ${ }^{1}$, Jonathan D. Buckley ${ }^{3}$, \\ Christopher K. Rayner ${ }^{1,2}$, Michael Horowitz ${ }^{1,2}$ and Karen L. Jones ${ }^{1,2 *}$ \\ ${ }^{1}$ Discipline of Medicine, Royal Adelaide Hospital, University of Adelaide, North Terrace, Adelaide, SA 5000, Australia \\ ${ }^{2}$ NHMRC Centre of Clinical Research Excellence in Nutritional Physiology, Interventions and Outcomes, Adelaide, SA 5000, \\ Australia \\ ${ }^{3}$ School of Health Sciences, Nutritional Physiology Research Centre and ATN Centre for Metabolic Fitness, University of South \\ Australia, Adelaide, SA 5000, Australia
}

(Received 20 September 2010 - Revised 19 January 2011 - Accepted 20 January 2011 - First published online 18 April 2011)

\begin{abstract}
In healthy older subjects, the glycaemic response to carbohydrate-containing meals is dependent on gastric emptying and intestinal absorption; when the latter is slowed, the magnitude of the rise in glucose is attenuated. The oligosaccharide $\alpha$-cyclodextrin has been reported to diminish the glycaemic response to starch in young adults; this effect has been attributed to the inhibition of pancreatic amylase. We examined the effects of $\alpha$-cyclodextrin on gastric emptying of, and the glycaemic and insulinaemic responses to, oral sucrose in healthy older subjects; as sucrose is hydrolysed by intestinal disaccharides, any effect(s) of $\alpha$-cyclodextrin would not be attributable to amylase inhibition. A total of ten subjects (seven males and three females, age 68-76 years) were studied on $2 \mathrm{~d}$. Gastric emptying, blood glucose and serum insulin were measured after ingestion of a $300 \mathrm{ml}$ drink containing $100 \mathrm{~g}$ sucrose, labelled with ${ }^{99 \mathrm{~m}} \mathrm{Tc}$-sulphur colloid, with or without $10 \mathrm{~g} \alpha$-cyclodextrin. Gastric emptying was slowed slightly by $\alpha$-cyclodextrin; this effect was evident between 135 and $195 \mathrm{~min}$ and was associated with a slight increase $(P<0.05)$ in distal stomach retention. After $\alpha$-cyclodextrin, blood glucose was slightly less $(P<0.05)$ at $60 \mathrm{~min}$, and serum insulin was less $(P<0.0005)$ at 90 and $120 \mathrm{~min}$. There was no difference in the incremental areas under the curve (iAUC) for blood glucose, but there was a trend for the iAUC for serum insulin to be lower $(P=0 \cdot 09)$ after $\alpha$-cyclodextrin. We conclude that in a dose of $10 \mathrm{~g}, \alpha$-cyclodextrin has modest effects to slow gastric emptying of, and modify the glycaemic and insulinaemic responses to, oral sucrose, probably due to delayed intestinal carbohydrate absorption.
\end{abstract}

Key words: Gastric emptying: Glycaemia: Cyclodextrin: Elderly

It is now well established that the rate of gastric emptying and small-intestinal carbohydrate absorption are major determinants of postprandial glycaemia in healthy subjects ${ }^{(1)}$ and patients with type $1^{(2)}$ and type 2 diabetes ${ }^{(3)}$, such that gastric emptying accounts for at least $35 \%$ of the variance in the initial rise in blood glucose, as well as influencing the incretin hormone (glucagon-like peptide-1 and glucose-dependent insulinotropic polypeptide) responses, after oral glucose ${ }^{(1,3,4)}$. Even relatively minor variations in the rate of gastric emptying can have a marked impact on the glycaemic response to carbohydrate $^{(5)}$, particularly as the relationship of glycaemia with small-intestinal carbohydrate delivery is non-linear ${ }^{(6)}$.

Cyclodextrins are cyclic oligosaccharides composed of six to eight glucose monomers ${ }^{(7)}$ that inhibit pancreatic amylase activity $^{(8)}$, are poorly digested in the small intestine ${ }^{(9)}$ and inhibit the hydrolysis of complex carbohydrates ${ }^{(8)} . \alpha$ - and $\beta$ Cyclodextrins have been reported to reduce the postprandial glycaemic $^{(7,8)}$, insulinaemic ${ }^{(7)}$ and glucose-dependent insulinotropic polypeptide responses ${ }^{(7)}$ to a starch meal. For example, in healthy young males, Raben et al. ${ }^{(7)}$ reported that peak blood glucose and plasma insulin and glucosedependent insulinotropic polypeptide concentrations were less when a potato starch meal was enriched with $2 \% \beta$-cyclodextrin, while plasma glucagon-like peptide-1 levels remained essentially unchanged. Subsequently, Buckley et al. ${ }^{(8)}$ reported, also in healthy subjects, that the area under the blood glucose curve after the consumption of white rice was reduced by approximately $50 \%$ by $10 \mathrm{~g}$ of $\alpha$-cyclodextrin.

Abbreviations: AUC, area under the curve; iAUC, incremental area under the curve.

*Corresponding author: Professor K. L. Jones, fax +61 88223 3870, email karen.jones@adelaide.edu.au 
A limitation of these studies ${ }^{(7,8)}$ was that gastric emptying was not measured; hence, it remains to be determined whether the observed effects on postprandial glycaemia were related to the effects on gastric emptying and/or intestinal glucose absorption.

The aims of the present study were to determine the effects of $\alpha$-cyclodextrin on gastric emptying of, and the glycaemic response to, an oral sucrose load in healthy older subjects. By selecting sucrose, a disaccharide hydrolysed by the intestine, rather than pancreatic enzymes, any effect(s) of $\alpha$-cyclodextrin would not reflect the inhibition of amylase. The broad hypothesis was that $\alpha$-cyclodextrin would attenuate the glycaemic response by slowing both gastric emptying and intestinal carbohydrate absorption.

\section{Materials and methods}

\section{Ethical approval and informed consent}

The present study was conducted according to the guidelines laid down in the Declaration of Helsinki, and all procedures were approved by the Research Ethics Committee of the Royal Adelaide Hospital (Adelaide, SA, Australia). Written informed consent was obtained from all subjects.

\section{Subjects}

In the present study, ten healthy older subjects (seven males and three females), with a median age of 70 (range 68-76) years and BMI of 26.9 (range $23 \cdot 2-32 \cdot 4$ ) $\mathrm{kg} / \mathrm{m}^{2}$ recruited by advertisement, were studied. The number of subjects studied was based on data from a previous study, which detected a reduction in the glycaemic response to a rice meal after $\alpha$ cyclodextrin when compared with the control ${ }^{(8)}$. All subjects were non-smokers, and none had a history of gastrointestinal disease or surgery, diabetes, significant respiratory, renal, hepatic or cardiac disease, chronic alcohol abuse or epilepsy, nor was taking medication known to influence gastrointestinal function.

\section{Protocol}

Each subject was studied on two occasions, on which they attended the Department of Nuclear Medicine, Positron Emission Tomography and Bone Densitometry at 08.30 hours following an overnight fast $(10.5 \mathrm{~h}$ for solids and $8.5 \mathrm{~h}$ for liquids) ${ }^{(10)}$. A cannula was placed in a left antecubital vein for blood sampling, and subjects were seated with their back against a gamma camera. Each subject rested comfortably in the sitting position for about $30 \mathrm{~min}^{(10)}$. At $t=-2 \mathrm{~min}$, subjects consumed a drink comprising $100 \mathrm{~g}$ sucrose dissolved in water (total volume of the drink $300 \mathrm{ml}$ ) and labelled with $20 \mathrm{MBq} 99 \mathrm{~m}$ Tc-sulphur colloid (Royal Adelaide Hospital Radiopharmacy, Adelaide, SA, Australia). On one of the days, $10 \mathrm{~g} \alpha$-cyclodextrin (Cavamax ${ }^{\circledR}$; Wacker Fine Chemicals, Adrian, MI, USA) were added to the sucrose before being dissolved in water (total volume $300 \mathrm{ml}$ ). The two studies were separated by at least $7 \mathrm{~d}$ and performed in a double-blind, randomised order. Gastric emptying, blood glucose and serum insulin were measured. At $t=300 \mathrm{~min}$, the intravenous cannula was removed, and subsequently, the subject was allowed to leave the laboratory. On both study days, subjects were given a light meal before leaving the laboratory.

\section{Measurements}

\section{Gastric emptying and intragastric distribution}

Subjects consumed the drink within $2 \mathrm{~min}$, and the time of drink completion was considered to be $t=0 \mathrm{~min}$. Radioisotopic data were acquired for $300 \mathrm{~min}$ (60) $\mathrm{s}$ frames for the first $60 \mathrm{~min}$ and $3 \mathrm{~min}$ frames thereafter ${ }^{(10)}$. Data were corrected for subject movement, radionuclide decay and $\gamma$-ray attenuation $^{(10)}$. Regions of interest were drawn around the total stomach, which was subsequently divided into the proximal and distal stomach regions, and gastric emptying curves (expressed as percentage retention over time) were derived. The amounts of the drink remaining in the total, proximal and distal stomach at $15 \mathrm{~min}$ intervals between $t=0$ and $300 \mathrm{~min}$ were calculated. The $50 \%$ gastric emptying time $\left(T_{50}\right)$ was also determined ${ }^{(10)}$.

\section{Blood glucose and serum insulin concentrations}

Venous blood samples (approximately $7.5 \mathrm{ml}$ ) were obtained immediately before the drink (i.e. $t=-2 \mathrm{~min}$ ) and at $15 \mathrm{~min}$ intervals between $t=0$ and $300 \mathrm{~min}^{(10)}$. Blood glucose concentrations were determined immediately using a portable blood glucose meter (Medisense Precision Q.I.D ${ }^{\mathrm{TM}}$ System, Abbott Laboratories; Medisense Products, Inc., Bedford, MA, USA) ${ }^{(10)}$. Blood samples for serum insulin were collected in ice-chilled serum tubes with clotting activator and stored at $-70^{\circ} \mathrm{C}$ for subsequent analysis, and insulin was measured on samples collected at $30 \mathrm{~min}$ intervals between $t=0$ and $300 \mathrm{~min}$. Insulin concentrations were measured by ELISA (Diagnostics Systems Laboratories, Inc., Webster, TX, USA). Sensitivity was $1.8 \mathrm{pmol} / 1$, intra-assay $\mathrm{CV}$ was $2.6 \%$ and inter-assay CV was $6 \cdot 2 \%{ }^{(5)}$.

\section{Statistical analysis}

Data were evaluated using repeated-measures two-way ANOVA, with 'treatment' and 'time' as within-subject factors. Gastric emptying, blood glucose and serum insulin concentrations were analysed as absolute values. Data were analysed from $t=0$ to $300 \mathrm{~min}$ to determine the effects ('treatment' and 'time') of sucrose and $\alpha$-cyclodextrin. In the event that the ANOVA demonstrated a statistically significant treatment $x$ time' interaction, post hoc tests were used to examine pointby-point comparisons between treatments, with significance corrected for multiple comparisons. In addition, based on our previous studies ${ }^{(1,3)}$, blood glucose concentration at 60 min was selected, a priori, for analysis. One-way ANOVA was used to analyse the effects of 'time' on blood glucose and serum insulin concentrations. Incremental areas under 
the curve (iAUC) were calculated using the trapezoidal rule for blood glucose and serum insulin from $t=-2$ to $300 \mathrm{~min}$. For gastric emptying (total, proximal and distal), total areas under the curve (AUC) were calculated from $t=0$ to $300 \mathrm{~min}$. All outcomes were analysed using paired $t$ tests. Relationships between variables were assessed using linear regression analysis. All analyses, unless otherwise stated, were performed using Statview (version 5.0; Abacus Concepts, Berkeley, CA, USA) and SuperANOVA (version 1.11; Abacus Concepts). iAUC and AUC analyses were performed by a professional statistician using SPSS version 18 (SPSS, Inc., Chicago, IL, USA). Data are presented as means with their standard errors. A $P$ value $<0.05$ was considered significant in all analyses.

\section{Results}

We recruited thirteen people, of which ten completed the study. Of the thirteen subjects, three were unwilling to continue with the study after experiencing diarrhoea on completion of the first visit. In the remaining subjects, the studies were generally well tolerated. Loose stools were reported by three of the ten subjects after $\alpha$-cyclodextrin (i.e. from $t=300 \mathrm{~min}$ ). In all cases, these symptoms were mild and had resolved spontaneously within $7 \mathrm{~h}$ of the completion of each study. No subject reported a difference in the taste of the drink between the study days.

\section{Gastric emptying}

Total stomach. On both study days, gastric emptying of glucose was non-linear and approximated a monoexponential pattern. There was a significant treatment $\times$ time interaction $(P<0.002)$ for gastric emptying so that intragastric content was slightly less $(P<0.05)$ between $t=135$ and $195 \mathrm{~min}$, after control, compared with $\alpha$-cyclodextrin. However, there was no difference between the AUC for gastric emptying after control than after $\alpha$-cyclodextrin $(P=0.22)$, nor any difference in the $T_{50}$ between the $2 \mathrm{~d}$ (control: 91.7 (SEM 10.7) min than after $\alpha$-cyclodextrin: 104.9 (SEM 19.4) min; $P=0 \cdot 37$; Fig. 1(a)).

Intragastric distribution. There was no difference in proximal stomach retention between the two study days (Fig. 1(b);
$P=0 \cdot 14)$ nor between the AUC for proximal retention after control than after $\alpha$-cyclodextrin $(P=0 \cdot 12)$. In contrast, there was a significant treatment $\times$ time interaction for the distal stomach $(P<0.005)$, so that retention of the drink was slightly greater after control than after $\alpha$-cyclodextrin at $t=90 \mathrm{~min}$ $(P<0.05)$. However, there was no significant difference between the AUC for distal stomach retention after control than after $\alpha$-cyclodextrin (Fig. 1(c); $P=0.79$ ).

\section{Blood glucose concentrations}

There was no significant difference in baseline (i.e. $t=-2 \mathrm{~min}$ ) blood glucose between the two study days (control $v$. $\alpha$-cyclodextrin: 6.3 (SEM 0.2$) \mathrm{mmol} / \mathrm{l}$ for both; $P=0.87$ ) (Fig. 2(a)). There was a rise in blood glucose after the drink on both days $(P<0.0001$ for both), which was evident from $t=15 \mathrm{~min}$ following both the control and $\alpha$-cyclodextrin $(P=0.0001$ for both). Peak blood glucose concentrations were not different after control $(10 \cdot 3$ (SEm 0.6$) \mathrm{mmol} / \mathrm{l}) v$. $\alpha$-cyclodextrin (10.3 (sem 0.5$) \mathrm{mmol} / \mathrm{l} ; \quad P=0.88)$. However, there was a significant 'treatment $\times$ time' interaction $(P<0.0001)$ for blood glucose concentrations so that at $t=60 \mathrm{~min}$, blood glucose was slightly greater $(P<0.05)$ and at $t=180$ and $210 \mathrm{~min}$ slightly less $(P<0.005)$ after control when compared with $\alpha$-cyclodextrin. There was no difference between the iAUC for blood glucose after control than after $\alpha$-cyclodextrin $(P=0.87)$. At $t=300 \mathrm{~min}$, there was a trend for blood glucose to be less than baseline after control $(P=0 \cdot 07)$, but not after $\alpha$-cyclodextrin $(P=0 \cdot 17)$.

\section{Serum insulin concentrations}

There was no significant difference in baseline (i.e. $t=-2 \mathrm{~min}$ ) serum insulin between the two study days (control $v$. $\alpha$-cyclodextrin: 66.7 (SEM 13.9) $v$. 72.9 (SEM 13.9) $\mathrm{pmol} / \mathrm{l} ; P=0.47$ ) (Fig. 2(b)). There was a rise in serum insulin after the drink on both days $(P<0.0001$ for both), which was evident from $t=30 \mathrm{~min}$ (the first time point at which insulin was measured) following both the control and $\alpha$-cyclodextrin $(P=0.0001$ for both). Peak serum insulin concentrations were not different after control (839 (SEM 150) pmol/l) than after $\alpha$-cyclodextrin (770.9 (SEM 137.5) $\mathrm{pmol} / \mathrm{l} ; \quad P=0 \cdot 22$ ). However, there was a significant
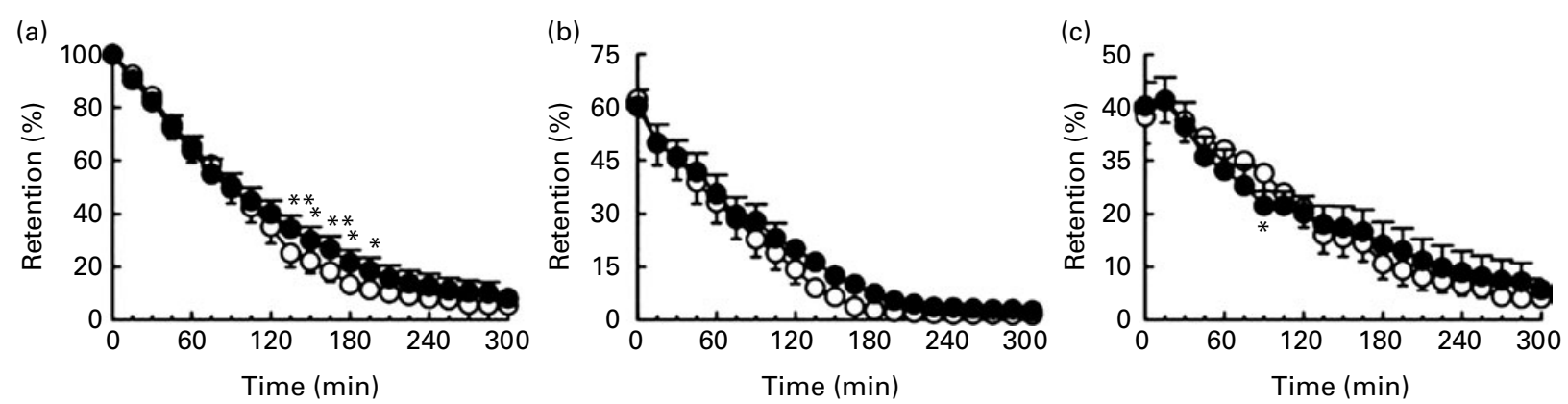

Fig. 1. Effects of control $(\mathrm{O})$ and $\alpha$-cyclodextrin $(10 \mathrm{~g})(\bullet)$ treatments on (a) total gastric emptying and (b) proximal and (c) distal stomach retention of a $300 \mathrm{ml}$ drink containing $100 \mathrm{~g}$ sucrose in ten healthy older subjects. Data are means, with their standard errors represented by vertical bars. Mean value was significantly different from that of the control treatment: ${ }^{\star} P<0.05,{ }^{\star \star} P<0.01$. 

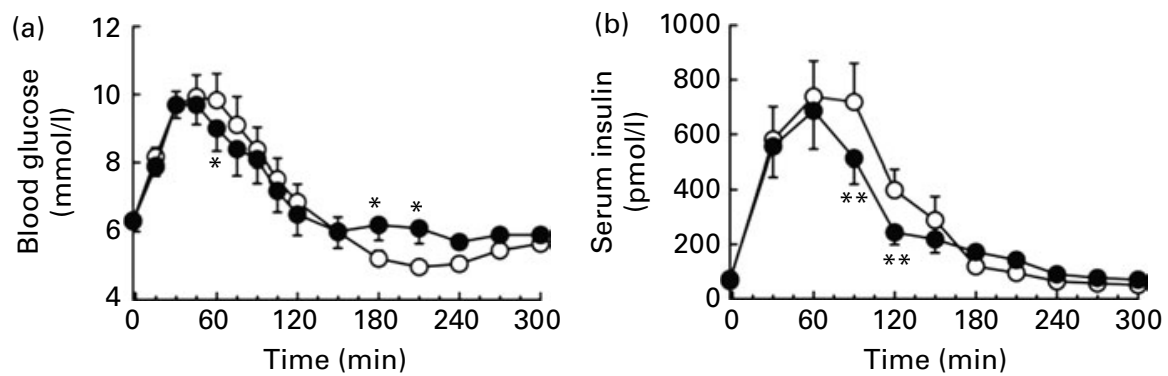

Fig. 2. Effects of control (o) and $\alpha$-cyclodextrin $(10 \mathrm{~g})(\bullet)$ treatments on (a) blood glucose and (b) serum insulin concentrations following a $300 \mathrm{ml}$ drink containing $100 \mathrm{~g}$ sucrose in ten healthy older subjects. Data are means, with their standard errors represented by vertical bars. Mean value was significantly different from that of the control treatment: ${ }^{\star} P<0.05,{ }^{\star \star} P<0.001$.

'treatment $\times$ time' interaction $(P<0.0001)$ for serum insulin concentrations. At $t=90$ and $120 \mathrm{~min}$, serum insulin was modestly greater $(P<0.0005)$ after control compared with $\alpha$-cyclodextrin. There was a trend for a significant difference between the iAUC for serum insulin after control than after $\alpha$-cyclodextrin $(P=0.09)$, representing a mean relative decrease in the iAUC of $17.0 \%$ after $\alpha$-cyclodextrin, compared with control. At $t=300 \mathrm{~min}$, there was no difference in serum insulin from baseline after control $(P=0 \cdot 26)$ or $\alpha$-cyclodextrin $(P=0 \cdot 81)$.

\section{Relationships between changes in blood glucose concentration with gastric emptying}

When data from the two study days were pooled, the magnitude of the rise in blood glucose from baseline was inversely related to the intragastric retention of the drink (e.g. percentage retention at $t=45 \mathrm{~min} ; r-0.46, P<0.04$; data not shown).

\section{Discussion}

The present study indicates that in healthy older subjects, $\alpha$-cyclodextrin, when given acutely in a dose of $10 \mathrm{~g}$, has modest effects to slow gastric emptying of, and modify the glycaemic and insulinaemic responses to, oral sucrose. Given that the digestion of sucrose is independent of the action of pancreatic amylase, these effects are not attributable to the inhibition of amylase.

$\alpha$-Cyclodextrin has been reported to attenuate the blood glucose $^{(7,8)}$ and serum insulin ${ }^{(7)}$ responses to starch meals, the digestion of which is dependent on amylase. When $\alpha$ cyclodextrin was included in the sucrose drink, we found that there was a slight lowering of blood glucose at 60 and $75 \mathrm{~min}$, and that blood glucose concentrations were subsequently slightly greater at 180, 195 and $210 \mathrm{~min}$. However, $\alpha$-cyclodextrin had no effect on the initial rise, peak blood glucose concentration or overall iAUC. In contrast to the present study, Raben et $a l .{ }^{(7)}$ and Buckley et $a l .{ }^{(8)}$ reported reductions in the iAUC for blood glucose of up to $50 \%$ in response to $\alpha$ cyclodextrin. The discordance between their observations ${ }^{(7,8)}$ and our own may reflect differences in the test meals and/or doses of $\alpha$-cyclodextrin used. In the present study, serum insulin was reduced by $\alpha$-cyclodextrin at 90 and $120 \mathrm{~min}$, and the observed initial lowering of glucose induced by $\alpha$-cyclodextrin, albeit small, is not accounted for by stimulation of insulin secretion. This is consistent with previous reports with starch meals that $\alpha$-cyclodextrin does not stimulate either insulin ${ }^{(8)}$ or incretin hormones postprandially ${ }^{(7)}$, and given that a sucrose drink was used, must be indicative of a minor reduction in the rate of small-intestinal glucose absorption. We have previously shown that modest variations in glycaemia have the potential to have a relatively greater effect on insulinaemia ${ }^{(1)}$. Hence, the reduction in the subsequent insulin response induced by $\alpha$-cyclodextrin may be attributable to the preceding decrease in glucose.

Gastric emptying of glucose, and other carbohydrates, is regulated at an overall rate of $4-17 \mathrm{~kJ} / \mathrm{min}$ as a result of a length-dependent inhibitory feedback from receptors located throughout the small intestine ${ }^{(11-13)}$. In the case of fat, protein and carbohydrate, it appears that this feedback is triggered by the digestion products, i.e. fatty acids, amino acids and monosaccharides $^{(14)}$. The observed effect of $\alpha$-cyclodextrin to slow gastric emptying, with concomitant changes in intragastric distribution, is novel. As in both healthy young and older subjects, perceptions of postprandial fullness ${ }^{(15)}$ and energy intake $^{(16)}$ are related to the content of the distal stomach (i.e. energy intake is less and fullness is greater when antral content is greater); this finding has potential implications for the regulation of appetite. Because differences in gastric emptying were only evident after approximately $135 \mathrm{~min}$, the observed slowing is unlikely to be mediated by changes in the physical characteristics of the intragastric content (viscosity) and probably reflects an increase in the smallintestinal feedback secondary to the exposure of a greater length of the small intestine to sucrose. The latter appears to be the primary mechanism by which guar gum ${ }^{(17,18)}$ and acarbose $^{(10)}$ slow gastric emptying. Given that the modest glucose-lowering effect was evident before any retardation of gastric emptying, we conclude that the former is likely to reflect primarily a delay in small-intestinal carbohydrate absorption, possibly due to the inhibitory effect of $\alpha$-cyclodextrin on the absorption of carbohydrate, so that carbohydrate is absorbed more distally. The viscous polysaccharide guar and some other fibres ${ }^{(19-21)}$ have similar effects. However, it should be recognised that even minor perturbations in the rate of gastric emptying can affect postprandial glycaemia substantially ${ }^{(5)}$. 
In interpreting our observations, it should be recognised that we utilised a dose $(10 \mathrm{~g})$ of $\alpha$-cyclodextrin that has been reported to diminish the glycaemic response to a carbohydrate (rice) meal in young, healthy subjects while inducing few, and tolerable, adverse gastrointestinal symptoms including nausea, abdominal bloating and diarrhoea ${ }^{(8)}$. While the reported effects of $10 \mathrm{~g}$ of $\alpha$-cyclodextrin on blood glucose were modest, this dose has been shown to reduce the blood glucose response to rice to a greater extent than $5 \mathrm{~g}^{(8)}$. It remains possible that a higher dose of $\alpha$-cyclodextrin may have induced greater effects on glycaemia and gastric emptying, but as three of the ten subjects reported adverse effects, it is likely that higher doses would not be well tolerated. It is also important not to extrapolate our observations to the effects of $\alpha$-cyclodextrin on the gastric emptying of starch meals where amylase inhibition is of major relevance. We would speculate that gastric emptying of starch may be accelerated initially as a result of reduced intestinal feedback, but that the effects on carbohydrate absorption, and hence glycaemia, may be greater.

\section{Acknowledgements}

The authors have no conflicts of interest or financial interests to declare. The present study was supported by a Royal Adelaide Hospital/Institute of Medical and Veterinary Science Research Committee Project Grant. D. G. was supported by a Postdoctoral Fellowship (PR 07A 3309) from the National Heart Foundation of Australia. K. L. J. was funded by a National Health and Medical Research Council of Australia Career Development Award. The authors wish to thank the staff of the Department of Nuclear Medicine, Positron Emission Tomography and Bone Densitometry, Royal Adelaide Hospital, for providing radiopharmaceuticals and gamma camera time, Amtrade International Private Limited (Adelaide, SA, Australia) and Wacker Chemicals (Mulgrave, VIC, Australia), for supplying $\alpha$-cyclodextrin, and Kylie Lange, Discipline of Medicine, University of Adelaide, for her involvement in the statistical analyses. The authors' contributions are as follows: D. G. was involved in the concept and design of the study, acquisition of subjects, data collection, analysis and interpretation, and preparation of the manuscript; L. V. and J. C. T were responsible for the data collection; J. M. W. performed the insulin analysis; J. D. B. and C. K. R. assisted in the preparation of the manuscript; M. H. and K. L. J. had a role in the design of the study, data analysis and interpretation, and preparation of the manuscript. Data from the present study have been presented in abstract form at the 19th IAGG World Congress of Gerontology and Geriatrics, Paris, France, 7 July $2009^{(22)}$.

\section{References}

1. Horowitz M, Edelbroek MA, Wishart JM, et al. (1993) Relationship between oral glucose tolerance and gastric emptying in normal healthy subjects. Diabetologia 36, $857-862$.
2. Ishii M, Nakamura T, Kasai F, et al. (1994) Altered postprandial insulin requirement in IDDM patients with gastroparesis. Diabetes Care 17, 901-903.

3. Jones KL, Horowitz M, Carney BI, et al. (1996) Gastric emptying in early noninsulin-dependent diabetes mellitus. $\mathrm{J} \mathrm{Nucl}$ Med 37, 1643-1648.

4. Nauck MA, Niedereichholz U, Ettler R, et al. (1997) Glucagon-like peptide 1 inhibition of gastric emptying outweighs its insulinotropic effects in healthy humans. Am J Physiol 273, E981-E988.

5. O'Donovan DG, Doran S, Feinle-Bisset C, et al. (2004) Effect of variations in small intestinal glucose delivery on plasma glucose, insulin, and incretin hormones in healthy subjects and type 2 diabetes. J Clin Endocrinol Metab 89, 3431-3435.

6. Pilichiewicz AN, Chaikomin R, Brennan IM, et al. (2007) Load-dependent effects of duodenal glucose on glycemia, gastrointestinal hormones, antropyloroduodenal motility, and energy intake in healthy men. Am J Physiol Endocrinol Metab 293, E743-E753.

7. Raben A, Andersen K, Karberg MA, et al. (1997) Acetylation of or beta-cyclodextrin addition to potato beneficial effect on glucose metabolism and appetite sensations. Am J Clin Nutr 66, 304-314.

8. Buckley JD, Thorp AA, Murphy KJ, et al. (2006) Dose-dependent inhibition of the post-prandial glycaemic response to a standard carbohydrate meal following incorporation of alpha-cyclodextrin. Ann Nutr Metab 50, 108-114.

9. Flourie B, Molis C, Achour L, et al. (1993) Fate of beta-cyclodextrin in the human intestine. J Nutr 123, 676-680.

10. Gentilcore D, Bryant B, Wishart JM, et al. (2005) Acarbose attenuates the hypotensive response to sucrose and slows gastric emptying in the elderly. Am J Med 118, 1289.

11. Brener W, Hendrix TR \& McHugh PR (1983) Regulation of the gastric emptying of glucose. Gastroenterology $\mathbf{8 5}, 76-82$

12. Lin HC, Doty JE, Reedy TJ, et al. (1989) Inhibition of gastric emptying by glucose depends on length of intestine exposed to nutrient. Am J Physiol 256, G404-G411.

13. Moran TH, Ladenheim EE \& Schwartz GJ (2001) Within-meal gut feedback signaling. Int J Obes Relat Metab Disord 25 , Suppl. 5, S39-S41.

14. Hunt JN (1980) A possible relation between the regulation of gastric emptying and food intake. Am J Physiol 239, G1-G4.

15. Jones KL, Doran SM, Hveem K, et al. (1997) Relation between postprandial satiation and antral area in normal subjects. Am J Clin Nutr 66, 127-132.

16. Sturm K, Parker B, Wishart J, et al. (2004) Energy intake and appetite are related to antral area in healthy young and older subjects. Am J Clin Nutr 80, 656-667.

17. Jones KL, MacIntosh C, Su YC, et al. (2001) Guar gum reduces postprandial hypotension in older people. $J \mathrm{Am}$ Geriatr Soc 49, 162-167.

18. Russo A, Stevens JE, Wilson T, et al. (2003) Guar attenuates fall in postprandial blood pressure and slows gastric emptying of oral glucose in type 2 diabetes. Dig Dis Sci 48, 1221-1229.

19. Holt S, Heading RC, Carter DC, et al. (1979) Effect of gel fibre on gastric emptying and absorption of glucose and paracetamol. Lancet i, 636-639.

20. Jenkins DJ, Wolever TM, Leeds AR, et al. (1978) Dietary fibres, fibre analogues, and glucose tolerance: importance of viscosity. Br Med J 1, 1392-1394.

21. Torsdottir I, Alpsten M, Holm G, et al. (1991) A small dose of soluble alginate-fiber affects postprandial glycemia and gastric emptying in humans with diabetes. J Nutr 121, 795-799.

22. Gentilcore D, Vanis L, Rayner CK, et al. (2009) Effects of $\alpha$-cyclodextrin on blood pressure, gastric emptying and glycaemia in the elderly. JNHA 13, Suppl. 1, S478. 